\title{
A RARE CASE OF PARAPARESIS
}

\author{
Dinesh Kumar1, Prasanth Varghese2, Percival Gilvaz ${ }^{3}$
}

${ }^{1}$ Senior Resident, Department of Neurology, Jubilee Mission Medical College, Trichur.

${ }^{2}$ Assistant Professor, Department of Neurology, Jubilee Mission Medical College, Trichur.

${ }^{3}$ Professor and HOD, Department of Neurology, Jubilee Mission Medical College, Trichur.

\section{ABSTRACT}

Neurological manifestations are common in HIV infection. They occur in all stages of HIV infection, early or late can affect any part of neuraxis. HIV associated myelopathy is a common neurological complication of AIDS; 20 to $55 \%$ of patients have evidence of spinal cord disease consistent with vacuolar myelopathy. ${ }^{1}$ The myelopathy usually manifests late in course of infection with slowly progressive weakness of lower extremities, gait disorder, sensory abnormalities in legs, impotency in men, urinary frequency and urgency. Here, we are presenting a case of HIV myelopathy in a 15-year-old boy with 6 months history of walking difficulty in form of pure motor progressive symmetric paraparesis. Patient managed on symptomatic measures and referred to HIV centre for further management.

\section{KEYWORDS}

Paraparesis.

HOW TO CITE THIS ARTICLE: Kumar D, Varghese P, Gilvaz P. A rare case of paraparesis. J. Evolution Med. Dent. Sci. 2016;5(69): 5047-5048, DOI: $10.14260 /$ jemds/2016/1146

\section{INTRODUCTION}

The course of spinal cord involvement in HIV is variable. It can lead to severe paralysis of lower limbs with loss of ability to walk and loss of sphincter control. The differential diagnosis is extensive and includes metabolic, infective and neoplastic spinal cord diseases. The diagnosis of this condition is mainly made on exclusion of other causes of myelopathy via serological, radiographic and other cerebrospinal fluid studies. The pathogenesis of vacuolar myelopathy is unknown. A metabolic disorder of vitamin B12 dependent transmethylation pathway induced by HIV or cytokine activation is considered the possible cause of vacuolar myelopathy associated with AIDS.

\section{CASE REPORT}

This 15-year-old boy presented with 6 months history of walking difficulty in form of dragging of both the lower limbs with occasional tripping and falls. He had no history of any upper limb or craniofacial symptoms. He also denied of sensory, cognitive symptomatology. His neurological examination showed weakness of both the lower limbs [grade $4+/ 5$ proximally, grade $4-/ 5$ distally]; the power in upper limb was normal. Beevor's sign was negative in this patient. He was also having spasticity confined to both the lower limbs. Mini mental status examination score was 30/30. The cranial nerve examination was within normal limits including vision and fundus. He had no sensory or cerebellar signs. In view of progressive pure motor symmetric paraparesis sparing bladder and without a spinal cord level, non-compressive myelopathy was considered. His MRI spine showed thin strip of white matter with hyperintensities confined to anterior part of spinal cord consistent with involvement of pyramidal tract.

Financial or Other, Competing Interest: None.

Submission 07-07-2016, Peer Review 12-08-2016,

Acceptance 20-08-2016, Published 29-08-2016.

Corresponding Author:

Dinesh Kumar,

F/27, Mata Kaulan Marg,

Kashmir Avenue,

Amritsar, Punjab.

E-mail: manishksharma27@gmail.com

DOI: 10.14260/jemds/2016/1146
His screening MRI brain showed significant white matter hyperintensities amounting to Fazeka grade 4. Radiological possibility entertained was leukodystrophy. However, his presentation was more acute and progressive since he was born of non-consanguineous parentally and there was no family history, acquired cause of similar presentation was looked into. During that evaluation he was found to be positive for HIV 1 tested by enzyme linked immunosorbent assay. CD4 count was 105 . So the final possibility considered was AIDS with HIV myelopathy.

\section{DISCUSSION}

The disease has predominant myelin loss in lateral and posterior column of spinal cord. ${ }^{1}$ The most common disease of spinal cord in HIV is the vacuolar myelopathy. ${ }^{1}$ The type of neurological involvement in HIV depends on stage of immune dysfunctioning. During early stages of immune dysfunction, manifestations like Bell's palsy and GBS are common, but during late stages more severe disorders like cryptococcal meningitis, TB meningitis, CMV encephalitis, HIV dementia and the polyradiculopathy appears. The virus damages the brain directly by invasion of CNS macrophages and glial cells or indirectly by release of neurotoxic cytokines, such as Interleukin beta, tumour necrosis factor alpha, Interleukin 6 and tumour growth factor beta. The myelopathy can be a cause of direct HIV infection or due to opportunistic infection like cytomegalovirus, HTLV 1 and HTLV 2.

The myelopathy in HIV can be of several types. The complications like mononeuritis multiplex can be there in HIV infections. Distal symmetrical sensory neuropathy is also seen in advanced stages of HIV. Autonomic neuropathy like postural hypotension and gastroparesis are also known to occur in HIV patients. The presentation of patients is usually in the form of length dependent neuropathy in form of burning feet and loss of pain, touch and temperature in glove in stocking distribution. The weakness of intrinsic foot muscles can be present in HIV associated neuropathy and ankle reflex may be absent. The necrosis of medial fasciculus gracilis is also found in some studies. ${ }^{2}$ Studies have shown association of raised vitamin B12 and folic acid levels in HIV myelopathy patients. The development of identifiable neurological syndrome in HIV is the culmination of a chain of 
events, determined by properties of HIV itself, genetic characteristics of host and interactions with environment. HIV is neuroinvasive, neurotropic and neurovirulent virus. ${ }^{3}$ HIV associated neurological syndrome can be classified as primary HIV neurological disease or secondary neurological disease.

In addition to its importance as a cause of neurological problems, HIV infection of CNS constitute a serious barrier to management and eradication of virus. The CNS is incompletely permeable to most of antiretroviral drugs. Axonal degeneration is a late presentation of vacuolar myelopathy in AIDS. Other than this the distal symmetrical sensory involvement of peripheral nerves is also an important neurological manifestation of HIV. The primary disorders result from direct effect of virus and include HIV associated neurocognitive disorder, HIV associated vacuolar myelopathy, distal sensory polyneuropathy. 4 The nervous system is frequently involved in HIV patients. ${ }^{5}$ Secondary disorders result from marked immuno-suppression and include opportunistic infections and primary central nervous system lymphoma.

The macrophages are the main cells infected with HIV and it seems that cytotoxic effects on the nervous tissue are indirect, may be due to release of substances by the infected macrophages. 5 The myeloradiculitis due to cytomegalovirus is a known complication in HIV patients with severe immunosuppression. Herpes simplex virus 2 myeloradiculitis is rare and often associated with disabling outcome. ${ }^{6}$ The imaging in the patients suffering from retrovirus mostly show contrast enhancement at conus medullaris and cauda equina. Herpes simplex virus 2 can also involve sacral region resulting in sacral myeloradiculitis with a fatal outcome in most of the patients. Myeloradiculitis due to herpes in retrovirus patients usually occurs in early stages.

\section{REFERENCES}

1. Di Rocco A. Diseases of spinal cord in human immunodeficiency virus infection. Semin Neurol 1999;19(2):151-5.

2. Petito CK, Vecchio D, Chen YT. HIV antigen and DNA in AIDS spinal cords correlate with macrophage infiltration not with vacuolar myelopathy. J Neuropathol Exp Neurol 1994;53(1):86-94.

3. Singer EJ, Valdes-Sueiras M, Commins D, et al. Neurologic presentations of AIDS. Neurol Clin 2010;28(1):253-75.

4. Bilgrami M, O'Keefe P. Neurologic diseases in HIV infected patients. Handb Clin Neurol 2014;121:1321-44.

5. Chimelli L, Rosemberg S. The neuropathology of the acquired immunodeficiency syndrome (AIDS). Rev Soc Bras Med Trop 1993;26(2):113-9.

6. Pinto A, Santos E, Correa DF, et al. CMV and HSV2 myeloradiculitis in HIV infected patient. Rev Inst Med Trop Sao Paulo 2011;53(3):173-5. 